\title{
Kognitive Verschlechterung wird verhindert
}

Fragestellung: Verringert eine multimodale Intervention mit Ernährungs-, Bewegungs- und kognitiven Trainingsmaßnahmen sowie Kontrolle von Gefäßrisikofaktoren die Verschlechterung kognitiver Fähigkeiten bei demenzgefährdeten älteren Menschen?

Hintergrund: Es besteht ein enger Zusammenhang von vaskulären und Lebensstilfaktoren mit Demenzerkrankungen, insbesondere auch der Alzheimer-Demenz (AD) [1]. Etwa ein Drittel der AD-Fälle kann mit sieben modifizierbaren Risikofaktoren in Verbindung gebracht werden [2].

Patienten und Methodik: Für die bevölkerungsbasierte randomisierte kontrollierte Doppelblindstudie wurden Teilnehmer

Ngandu T, Lehtisalo J, Solomon $A$ et al. A 2 year multidomain intervention of diet, exercise, cognitive training, and vascular risk monitoring versus control to prevent cognitive decline in at-risk elderly people (FINGER): a randomized controlled trial. Lancet. 2015; 385: 2255 - 63 früherer nationaler Umfragen im Alter von 60 bis 70 Jahren rekrutiert, die einen Risikoscore (Cardiovascular Risk Factors, Aging and Dementia) von mindestens sechs Punkten und ein etwa altersdurchschnittliches kognitives Niveau aufweisen mussten. Die Randomisie- rung erfolgte entweder zur multimodalen Intervention oder zur Kontrollbedingung. Die Intervention umfasste eine angepasste Ernährung, regelmäßiges körperliches wie kognitives Training und Messungen kardiovaskulär relevanter Parameter. Primärer Zielparameter war der z-Wert auf einer standardisierten neuropsychologischen Testbatterie (NTB) nach zwei Jahren.

Ergebnisse: 591 Teilnehmer der Interventions- und 599 der Kontrollgruppe konnten ausgewertet werden. Die mittlere Änderung hinsichtlich des primären Endpunkts betrug 0,20 \pm 0,51 in der Interventions- und 0,16 $\pm 0,51$ in der Kontrollgruppe. Der Unterschied im NTB-Gesamtscore war signifikant mit 0,022 (95\%-Konfidenzintervall 0,002-0,042, $\mathrm{p}=0,030$ ). Die Verbesserung der globalen Kognition in beiden Gruppen war in der Interventionsgruppe um $25 \%$ größer. Bei den exekutiven Funktionen kam es sogar zu einer Verbesserung um $83 \%(\mathrm{p}=0,039)$, bei der kognitiven Verarbeitungsgeschwindigkeit um $150 \%$ $(\mathrm{p}=0,029)$. Die Gedächtnisleistung war nicht signifikant besser.

Schlussfolgerungen: Eine zweijährige multimodale Intervention aus Ernährungs-, Bewegungs-, kognitiven Trainingsmaßnahmen und die Kontrolle vaskulärer Risikofaktoren könnte zum Erhalt oder zur Verbesserung der kognitiven Leistungsfähigkeit in einer Risikopopulation für Demenzerkrankungen führen.

\section{- Kommentar von Christian Lange-Asschenfeldt, Düsseldorf}

\section{Gefäßprävention auch wirksam bei der Alzheimer-Demenz}

Der Wert präventiver Maßnahmen, insbesondere im Hinblick auf das kardiovaskuläre Risiko, ist bei Demenzerkrankungen durch Beobachtungs- und Interventionsstudien gesichert. Aufgrund der erheblichen Überlappung der Risikofaktoren gilt dies nicht nur für vaskuläre Demenzen oder Mischformen mit vaskulären Anteilen, sondern ausdrücklich auch für die AD als primär neurodegenerative Demenz. Dies liegt unter anderem daran, dass es bereits früh im Verlauf der AD auch zu funktionellen und morphologischen Endothelveränderungen (z.B. durch Amyloidablagerung) kommt. Bei ohnehin vorgeschädigten Hirngefäßen ist dann die „vaskuläre Reserve" geringer und zusätzlich zu den AD-spezifischen Veränderungen treten hypoxische Schäden auf, die den neurodegenerativen Prozess beschleunigen, was den Eintritt in einen Circulus vitiosus bedeutet $[3,4]$. Auch ist endotheliale Integrität in Hirngefäßen ein Faktor bei der Clearance von $\beta$-Amyloid aus dem ZNS und wirkt so dem neurodegenerativen Prozess bei der AD entgegen.

Ngandu et al. weisen nach, dass ein positiver Effekt eines "breiten Angriffs" mit multimodaler Intervention bevölkerungsbasiert in einem Public-Health-Kontext nachweisbar ist. Die Studie ist methodisch sehr gut gemacht, was angesichts des immensen Aufwands, den eine moderne mehrjährige bevölkerungsbasierte Präventionsstudie bedeutet, kaum genug zu würdigen ist. Geringe Effektstärken (wie hier) haben in solchen großen bevölkerungsbasierten Langzeitstudien zur Prävention häufiger Erkrankungen dennoch möglicherweise eine hohe Relevanz. Geplant ist eine weitere Verlaufsbeobachtung über sieben Jahre, um auch konkrete Effekte auf die Inzidenz von Demenzerkrankungen und speziell der AD zu ermitteln. Zu untersuchen wäre natürlich auch, zu welchem Grad die einzelnen Interventionen zum Gesamteffekt beitragen.
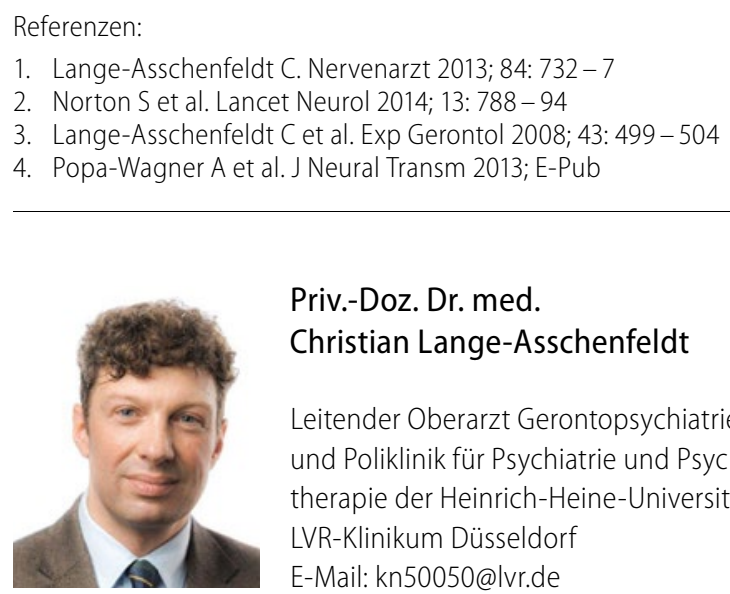

Priv.-Doz. Dr. med. Christian Lange-Asschenfeldt

Leitender Oberarzt Gerontopsychiatrie, Klinik und Poliklinik für Psychiatrie und Psychotherapie der Heinrich-Heine-Universität LVR-Klinikum Düsseldorf E-Mail:kn50050@lvr.de 